\title{
How Negative Emotion Enhances the Visual Specificity of a Memory
}

\section{Citation}

Kensinger, Elizabeth A., Rachel J. Garoff-Eaton, and Daniel L. Schacter. 2007. How negative emotion enhances the visual specificity of a memory. Journal of Cognitive Neuroscience 19(11): 1872-1887.

\section{Published Version}

doi:10.1162/jocn.2007.19.11.1872

\section{Permanent link}

http://nrs.harvard.edu/urn-3:HUL.InstRepos:3622259

\section{Terms of Use}

This article was downloaded from Harvard University's DASH repository, and is made available under the terms and conditions applicable to Other Posted Material, as set forth at http:// nrs.harvard.edu/urn-3:HUL.InstRepos:dash.current.terms-of-use\#LAA

\section{Share Your Story}

The Harvard community has made this article openly available.

Please share how this access benefits you. Submit a story.

\section{Accessibility}




\title{
How Negative Emotion Enhances the Visual Specificity of a Memory
}

\author{
Elizabeth A. Kensinger ${ }^{1,2}$, Rachel J. Garoff-Eaton ${ }^{2,3}$, \\ and Daniel L. Schacter ${ }^{2,3}$
}

\begin{abstract}
Some studies have suggested that emotion primarily increases memory for "gist," and does not enhance memory for detail. There are, however, some instances in which negative objects (e.g., snake, grenade) are remembered with more visual detail than neutral objects (e.g., barometer, blender). In the present functional magnetic resonance imaging (fMRI) study, we examined the encoding processes that lead a person to remember the exact visual details of negative and neutral objects, and to remember which of two decisions were made about the objects (a size decision or an animacy decision). The enhancement in memory for a negative item's visual details
\end{abstract}

\section{INTRODUCTION}

Individuals often claim to remember emotional experiences with rich detail. Laboratory studies repeatedly have demonstrated that people are more likely to report vivid recollections of emotional events than of nonemotional ones (e.g., Kensinger \& Corkin, 2003; Dewhurst \& Parry, 2000; Ochsner, 2000). Despite the subjective vividness associated with emotional experiences, however, numerous studies have revealed that emotion does not enhance memory for all types of detail. Rather, emotion often has focal effects on memory, with only some aspects of an experience more likely to be remembered because of its emotional content (see Reisberg \& Heuer, 2004; Buchanan \& Adolphs, 2002 for recent reviews). For example, Adolphs and colleagues have shown that emotion can enhance memory for the "gist" or general theme of presented information, but can reduce memory for details (Denburg, Buchanan, Tranel, \& Adolphs, 2003; Adolphs, Denburg, \& Tranel, 2001). They also have provided evidence that these mnemonic effects are mediated by the amygdala: Individuals with amygdala damage do not show these gist-enhancing and detail-impairing effects of emotion on memory (Adolphs, Tranel, \& Buchanan, 2005).

\footnotetext{
${ }^{1}$ Boston College, ${ }^{2}$ Athinoula A. Martinos Center for Biomedical Imaging, Charlestown, MA, ${ }^{3}$ Harvard University
}

appeared to result from enhanced visual processing: The right fusiform gyrus, a region known to be critical for processing exemplar-specific details, showed a greater extent and magnitude of activity during the successful encoding of negative objects. Activity in the right amygdala also corresponded with memory for visual detail, although it did not relate to memory for the task performed with the item. These data provide strong evidence that engagement of some amygdalar regions can correspond with enhanced memory for certain types of details, but does not ensure successful encoding of all contextual details.

Not all effects of emotion on memory, however, are explained by this gist versus detail framework. Emotion sometimes can enhance the visual specificity with which information is remembered: Individuals are better at distinguishing "same" from "similar" object exemplars when those exemplars are negative than when they are neutral (Kensinger, Garoff-Eaton, \& Schacter, 2006, 2007), suggesting that individuals are more likely to remember exactly what a negative object looked like when presented at study.

The primary goal of the present study was to examine the neural processes that lead to this enhancement in memory for the visual details of negative objects. A growing number of neuroimaging studies have attempted to elucidate the neural processes that contribute to memory specificity for neutral items (for reviews, see Schacter, Gallo, \& Kensinger, 2007; Schacter, Dobbins, \& Schnyer, 2004), but there is a virtual absence of such research concerning emotionally arousing stimuli. Prior research examining participants' abilities to remember the visual details of neutral items has revealed a critical role of the right fusiform gyrus. Activity in the right fusiform gyrus corresponds with subsequent memory for the exact visual details of a studied object (e.g., being able to identify not only that an umbrella had been studied, but also to identify which particular umbrella had been studied; Garoff, Slotnick, \& Schacter, 2005), consistent with previous findings suggesting that the right fusiform gyrus is important for the processing of 
the exemplar-specific visual details of an object (Simons, Koutstaal, Prince, Wagner, \& Schacter, 2003; Koutstaal et al., 2001; Marsolek, 1999). Based on this prior literature, we hypothesized that activity in the right fusiform gyrus would correspond with enhanced visual specificity for negative objects as well as for neutral ones.

The critical question was whether amygdala activity at encoding also would correspond with memory for visual detail. The amygdala often has been shown to modulate sensory functioning (Dolan \& Vuilleumier, 2003; Davis \& Whalen, 2001; LeDoux, 1995). Individuals are more likely to detect emotional than neutral objects in visual arrays (Ohman, Flykt, \& Esteves, 2001) or to identify emotional compared to neutral stimuli presented only briefly (Phelps, Ling, \& Carrasco, 2006; Anderson \& Phelps, 2001), and recent neuroimaging data have suggested that these effects are due to interactions between the amygdala and the fusiform gyrus (e.g., Noesselt, Driver, Heinze, \& Dolan, 2005; Vuilleumier, Richardson, Armony, Driver, \& Dolan, 2004) and between the amygdala and the occipital cortex (e.g., Tabert et al., 2001). Although these studies have shown a link between amygdala activity and sensory processing, they have not revealed whether connections between the regions influence the visual specificity of a memory. In fact, there has been significant debate about whether amygdala engagement would correspond with enhanced or reduced visual specificity: Although it might seem intuitive that such connections would enhance visual specificity, a couple of studies have shown stronger relations between activity in visual processing regions and the successful encoding or retrieval of neutral as compared to emotional information (Dolcos, LaBar, \& Cabeza, 2004; Sharot, Delgado, \& Phelps, 2004). Although these studies have not examined the visual specificity of a memory, they raise the intriguing possibility that the ability to remember visual information may be reduced, rather than enhanced, by emotion. Moreover, studies by Adolphs et al. (2005) have suggested that amygdala engagement may impair, rather than enhance, the ability to encode specific visual details (see review by Buchanan \& Adolphs, 2002). However, our study differed from those of Adolphs and colleagues in an important way: In the present study, participants were asked to remember the visual details of the emotional items themselves (e.g., to remember what a snake looked like), whereas in the studies by Adolphs and colleagues (e.g., Adolphs et al., 2001, 2005; Denburg et al., 2003), participants often were asked to remember the details of information peripheral to the emotional content of the scene or story (e.g., to remember where on the branch the snake was perched, or what the forest looked like). We have shown previously that individuals are better able to remember the visual details of negative objects than of neutral ones (Kensinger et al., 2006, 2007), and we hypothesized that amygdala-fusiform interactions would be likely to underlie the enhancing effects of negative emotion on memory specificity.
The secondary goal of the present study was to examine the mechanisms through which negative emotion exerts focal effects on memory. Prior studies have revealed that amygdala activity does not always correspond with subsequent memory for details: Although amygdala activity is associated with the ability to remember whether a negative item was seen or imagined (Kensinger \& Schacter, 2005a, 2005b), it does not relate to the ability to remember which decision was made about a negative or positive item (Kensinger \& Schacter, 2006a). We expected to replicate within a single study these focal effects of amygdala activity on memory for detail. We hypothesized that amygdala activity would be related to the encoding of details that are intrinsically linked to the negative item itself (e.g., its physical appearance) but not to elements peripheral or extrinsic to the item (e.g., the task performed with the item; see Mather et al., 2006; Kensinger \& Schacter, 2006a for further discussion).

By assessing memory for two different types of detail within the same experiment, the current study also could examine whether there were particular neural processes that corresponded both with the ability to remember intrinsic details of a negative item and also with the inability to remember an extrinsic detail of that item's presentation. To the extent that the focal effects of emotion on memory arise from encoding processes (e.g., attention toward features directly tied to the emotional item), we would expect to see activity that would both facilitate the successful encoding of the intrinsic details of an emotional item and impede encoding of a more extrinsic detail regarding the item's presentation. An open question was whether this activity would be within the standard fronto-parietal network that guides attention toward any task-relevant stimuli, or whether this network would instead include regions specifically tuned to the processing of emotional information (e.g., orbito-frontal cortex).

\section{METHODS}

\section{Participants}

Twenty-one native English-speaking adults participated in this study. The data from one participant were excluded due to scanner malfunction. The remaining 20 participants (10 women) ranged in age from 19 to 31 years (mean age $=22$ years). All participants had normal or corrected-to-normal vision, and all were screened to exclude those with any contraindicators for MRI scanning, or with any history of neurological trauma or psychiatric disorder. No participant reported taking any medications that affected the central nervous system. Informed consent was obtained from all participants in a method approved by the Harvard University and Massachusetts General Hospital Institutional Review Boards. 


\section{Materials and Procedure}

Materials comprised 386 pairs of colored, nameable photo objects (Hemera Technologies, 2002, Canada), sized to 300 pixels in their largest dimension. Pairs of objects were selected so that the two items of a pair shared the same verbal label (e.g., were both umbrellas) but differed in other perceptual features (e.g., color, shape, size, orientation). Object pairs were those used in Kensinger et al. (2006); as described in that paper, half of the pairs had been rated by participants as negative and arousing, and half had been rated as neutral and nonarousing. Pairs also had been selected to assure that the negative and neutral item pairs were matched for the overall similarity of the two items, the dimensions (color, size, shape, orientation) that differed between the two items, and the familiarity of the items.

\section{Study Procedure}

While in the functional magnetic resonance imaging (fMRI) scanner, participants were presented with 304 nameable, colored objects. These objects were presented across four lists, with each list containing 76 items (half negative, half neutral). Each item was presented for $1 \mathrm{sec}$. For half of the items, participants made a size decision about whether each object, in the real world, would fit inside a filing cabinet drawer. For the other half of the objects, participants decided whether the object was a living thing. Participants were cued as to which decision to make by the prompts "Living?" or "Fit?". These prompts appeared above the object. Participants were asked to make their decision as quickly as possible. There were no reaction time differences in the time it took participants to make the decision for the negative and neutral objects. Following the item's presentation, a fixation cross $(+)$ was presented for a variable duration (range of 5-13 sec) to provide jitter (Dale, 1999).

\section{Test Procedure}

Outside of the scanner, after approximately a 30-min delay, participants performed a surprise object recognition task. On the recognition task, participants were presented with 380 objects: 152 objects (76 negative, 76 neutral) were identical to those that had been studied (same objects), 152 objects (76 negative, 76 neutral) shared the same verbal label as a studied item but that differed in color, size, shape, or orientation (similar objects), and 76 objects were new (38 negative, 38 neutral; Figure 1). Each object appeared in the center of the computer screen, with a prompt below indicating that participants should indicate, by keypress, whether the item was "same," "similar," or "new."

When participants gave a "same" or a "similar" response to an item on the recognition memory task, they were indicating that they had seen that type of item before (e.g., that they had seen a toaster on the study list). In these instances, the participants were then asked to indicate which task they had performed with the item (i.e., whether they had decided if the toaster was living or if the toaster would fit in a drawer). It is reasonable for participants to know that they saw a particular type of item (e.g., a toaster) and to know the task that they performed with that item (e.g., that they decided whether the toaster would fit in a drawer) and yet to be incorrect about the visual details of that toaster (e.g., to give a "similar" rather than a "same" response to a same toaster). Conversely, participants can be correct about the visual details of a studied item (e.g., can correctly give a "same" response to a same toaster) and yet can be incorrect about the task performed with the item. When participants gave a "new" response, they were indicating no memory for that type of object. Thus, in instances where a "new" response was given, participants were not asked to indicate what task they had performed with the item (because, by definition, a "new" response meant that the participant believed that they had never studied the item).

The member of the object pair that was included on the recognition task was held constant for all participants; the items presented at study and the task performed with each study item were counterbalanced between participants to manipulate the condition of each object shown at recognition. An equal proportion of studied items was tested as same and as similar exemplars to avoid biasing participants' responses to the test exemplars. However, the neuroimaging analyses focus on participants' encoding of items that were later tested with the same exemplar. Participants' responses to same items are straightforward to interpret: A "same" response to a same item reflects memory for the specific visual details of a studied item (i.e., the ability to know that the exemplar tested is exactly the same exemplar that was studied); a "similar" response indicates memory for the general type of item but not for its exact visual details (otherwise, the participant should know that the test exemplar is the "same" as the studied one); and a "new" response reflects complete forgetting of the item's presentation. Task-correct or task-incorrect decisions also are clear-cut in the case of the same items. Either a person correctly remembers the task performed with the studied item, or he does not. In contrast, interpretation of responses to items tested as similar exemplars is more difficult to interpret; for example, a "similar" response to a similar exemplar could reflect memory for specific visual details (e.g., a participant could remember exactly what the studied umbrella looked like, and therefore could know that the tested exemplar is not the same) or it could reflect memory for only the general item type (e.g., a participant could remember that an umbrella was studied but, having no memory for its visual details, indicate that the umbrella was "similar" to the studied one). It also is more difficult 


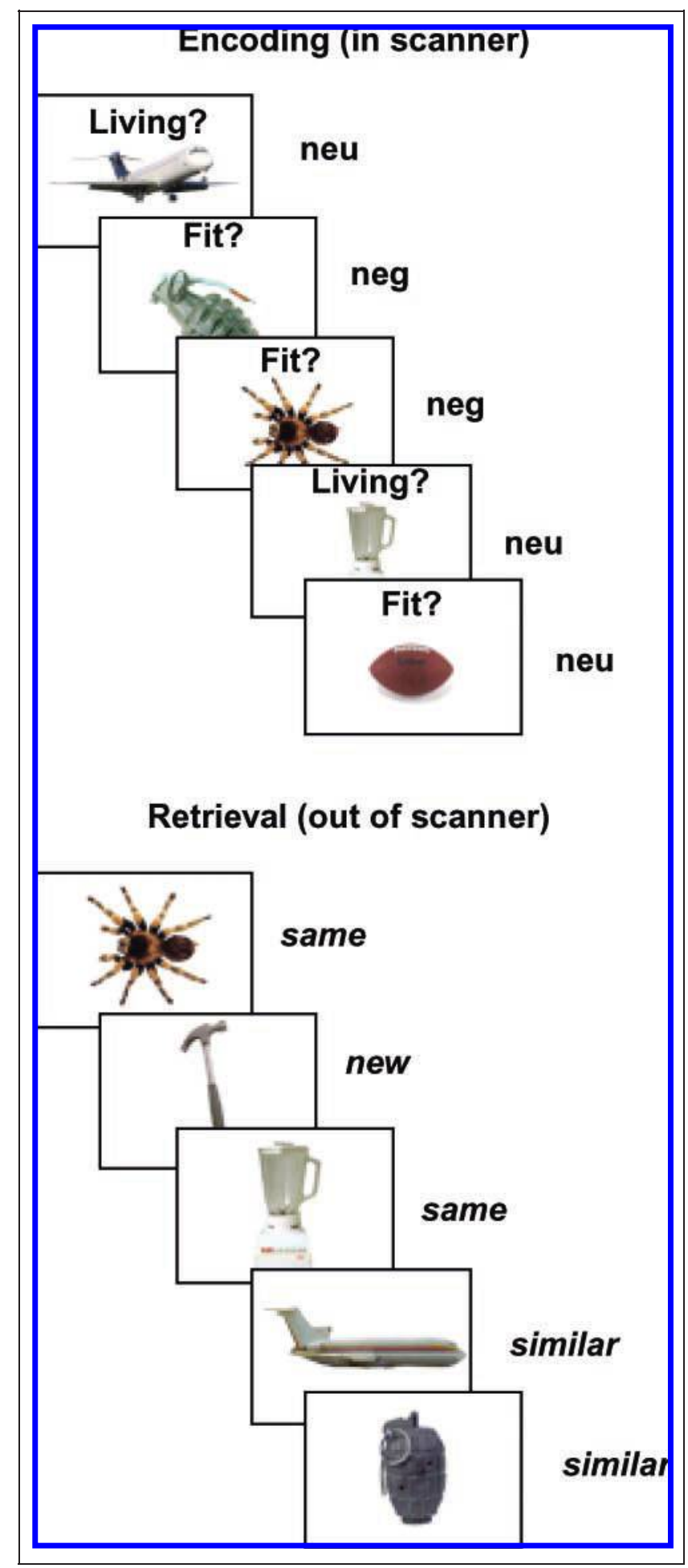

Figure 1. At encoding, participants saw negative and neutral objects, each presented for $1 \mathrm{sec}$. They were asked to judge either whether the object would fit inside of a filing cabinet drawer or whether the object was living. At retrieval, participants saw some objects that were identical to a studied item (same), others that shared the same verbal label but that were a different exemplar of the item (similar), and others that had not been studied (new). Half of the same and similar objects had been studied with the fit task, and half had been studied with the living task. Participants indicated whether the object was "same," "similar," or "new," and for items judged to be "same" or "similar," participants indicated which of the two judgments had been made about the item (fit or living). to interpret task-correct and task-incorrect assignments for the similar exemplars because, in reality, the person was never asked to perform any task with that exact exemplar.

\section{Image Acquisition and Data Analysis}

Images were acquired on a 3-Tesla Siemens Allegra MRI scanner. Detailed anatomic data were acquired using a multiplanar rapidly acquired gradient-echo (MP-RAGE) sequence. Functional images were acquired using a T2*weighted echo-planar imaging (EPI) sequence $(\mathrm{TR}=$ $2000 \mathrm{msec}, \mathrm{TE}=30 \mathrm{msec}, \mathrm{FOV}=200 \mathrm{~mm}$; flip angle $=$ $90^{\circ}$ ). Twenty-eight axial-oblique slices (3.2 mm thickness, $0.6 \mathrm{~mm}$ skip between slices), aligned along the anterior commissure/posterior commissure line, were acquired in an interleaved fashion.

All preprocessing and data analysis were conducted within SPM99 (Wellcome Department of Cognitive Neurology). Standard preprocessing was performed on the functional data, including slice-timing correction, rigid body motion correction, normalization to the Montreal Neurological Institute template (resampling at $3 \mathrm{~mm}$ cubic voxels), and spatial smoothing (using an 8-mm full-width half maximum isotropic Gaussian kernel).

For each participant, and on a voxel-by-voxel basis, an event-related analysis was first conducted in which all instances of a particular event type were modeled through convolution with a canonical hemodynamic response function. All participants had at least 15 instances of every modeled event type. Effects for each event type were estimated using a subject-specific, fixedeffects model. These data were then entered into a second-order, random-effects analysis.

Random-effects contrast analyses contrasted activation as a function of subsequent memory performance (e.g., later remembered with detail, later remembered without detail, later forgotten) separately for each emotion type (negative or neutral) and for each type of detail being assessed (memory for visual details or memory for task performed). Conjunction analyses (using the masking function in SPM) were then used to examine what regions showed activation across two or more contrasts, and interaction analyses were performed to reveal what regions showed a greater correspondence to subsequent memory performance for one emotion type than for the other. All activations are presented in neurological coordinates (i.e., activity on the right hemisphere is presented on the right side of the brain images). Voxel coordinates are reported in Talairach coordinates (Talairach \& Tournoux, 1988) and the center voxel was identified within the cluster of activation.

Event-related time-courses were extracted from active clusters by creating regions of interest (ROI), including all significant voxels within an $8-\mathrm{mm}$ radius of each chosen maximum voxel, using the ROI toolbox implemented in SPM (written by Russell Poldrack). Within 
Table 1. Proportion [mean $(S E)$ ] of Items Given a "Same," "Similar," or "New" Response as a Function of Item Type (Same, Similar, or New) and Emotion Type (Neutral or Negative Arousing)

\begin{tabular}{lccc}
\hline Response & Same & Similar & New \\
\hline Neutral & & & \\
"Same" & $0.46(0.02)$ & $0.14(0.03)$ & $0.02(0.02)$ \\
"Similar" & $0.29(0.02)$ & $0.41(0.04)$ & $0.19(0.04)$ \\
"New" & $0.25(0.03)$ & $0.45(0.03)$ & $0.79(0.03)$ \\
& & & \\
Negative Arousing & & & \\
"Same" & $0.51(0.02)$ & $0.14(0.05)$ & $0.03(0.02)$ \\
"Similar", & $0.26(0.04)$ & $0.44(0.03)$ & $0.20(0.03)$ \\
"New", & $0.22(0.03)$ & $0.42(0.02)$ & $0.77(0.03)$ \\
\hline
\end{tabular}

each of these ROIs, a hemodynamic response function was calculated for each individual subject and for each condition type (relative to fixation baseline) as a function of peristimulus time $(0-20 \mathrm{sec})$. Statistics were performed on the sum of the signal change within peristimulus times 4-10 sec. These signal change values are displayed in the figures. Analysis of variance (ANOVA) was performed on these extracted signal change values to examine whether the ROIs showed an interaction between memory accuracy and emotion type (e.g., activity relating to memory for the details of an emotional item but not for details of a neutral item, or vice versa), between memory accuracy and detail type (e.g., activity relating to memory for visual detail but not to memory for the task performed, or vice-versa), or among all three factors.

\section{RESULTS}

Both behavioral and neuroimaging data were analyzed with participants' sex as a factor. No effects of sex were revealed, and so all reported analyses collapse together data from men and women.

\section{Behavioral Results}

As in previous studies using variants of this paradigm (Kensinger et al., 2006; Garoff et al., 2005), we considered "same" responses to a same studied item to reflect memory for specific visual details ("specific recognition") and "similar" responses to a same studied item to indicate memory for some (but not all) aspects of the studied item ("nonspecific recognition"). "New" responses to same items, in contrast, reflected a complete miss (i.e., participants did not remember that the particular type of item had previously been studied; refer to Methods for a more detailed discussion of the study design logic). Consistent with a prior behavioral study
(Kensinger et al., 2006), negative items were more likely to be remembered with specific visual detail than were neutral items. Thus, specific recognition rates (saying "same" to a same item) were significantly higher for negative items (51\%) than for neutral items [46\%, $t(19)=$ $3.58, p<.01$; see Table 1 ]. No effects of emotion were noted for responses given to similar or new items $(p>$ .15). Also consistent with a prior study (Kensinger \& Schacter, 2006a), the likelihood of remembering the task performed with the item (whether it would fit in a drawer or whether it was a living thing) was not affected by the emotional content of the item $(p>.15$; see Table 2).

It is important to note that participants' performance on the task was good, despite the large number of studied items. If performing at chance, participants would have been equally likely to give "same," "similar," or "new" responses to same items, and they would have given each type of response $33 \%$ of the time. Instead, participants gave "same" responses to same items significantly more than $33 \%$ of the time. Moreover, they were least likely to call a same item "new," were somewhat more likely to call a same item "similar," and were most likely to call a same item "same." Thus, the large number of studied items allowed us to analyze all responses to same items (i.e., resulted in a sufficient number of "new," "similar," and "same" responses to same items), but did not lead to memory performance that approached chance.

\section{Subsequent-Memory fMRI Results}

If we consider "same" and "similar" responses to same items to reflect at least some memory for the studied items, and "new" responses to same items to reflect forgetting of the studied item, then comparing ("same" + "similar") > "new" responses to same items gives us a way to examine the standard subsequent-memory effect (i.e., later remembered $>$ later forgotten) for the emotional and the neutral items. We performed a conjunction analysis to examine the regions that showed this

Table 2. Proportion [mean $(S E)$ ] of Items Attributed to the Correct Study Task as a Function of Item Type (Same, Similar), Response Type ("Same," "Similar"), and Emotion Type (Neutral, Negative Arousing)

\begin{tabular}{lcc}
\hline Response & Same & Similar \\
\hline Neutral & & \\
"Same" & $0.66(0.02)$ & $0.65(0.04)$ \\
"Similar", & $0.58(0.03)$ & $0.62(0.03)$ \\
Negative Arousing & & \\
"Same" & $0.68(0.03)$ & $0.70(0.03)$ \\
"Similar", & $0.61(0.04)$ & $0.66(0.02)$ \\
\hline
\end{tabular}


subsequent-memory effect for both the negative and neutral items. We created a mask of the regions revealed in the contrast of subsequent ("same" + "similar") > "new" responses to same neutral items and then applied that mask to the contrast of subsequent ("same" + "similar") > "new" responses to same negative items to reveal only regions that were active in both contrasts. The individual contrasts included in the conjunction analysis were analyzed at a threshold of $p<.01$, uncorrected and with a 5-voxel extent, so that the conjoint probability of the conjunction analysis was $p<.001$, uncorrected (Lazar, Luna, Sweeney, \& Eddy, 2002; Fisher, 1950). We also performed a whole-brain interaction analysis to examine the regions that showed a stronger relation to subsequent-memory for the negative items than for the neutral items [i.e., ("same" + "similar") > "new" to same negative items $>$ ("same" + "similar") $>$ "new" to same neutral items]. This interaction analysis was conducted at a standard threshold of $p<.001$ and revealed regions with at least a 5-voxel extent. As reported in Table 3, we found a fronto-temporal network of regions that related to memory for negative arousing and neutral items (consistent with regions implicated in many subsequent-memory paradigms; see Paller \& Wagner, 2002 for review) and we found that activity in the amygdala, ventrolateral/orbito-frontal prefrontal cortex, hippocampus, and parahippocampal gyrus showed a stronger relation to subsequent memory for the negative arousing items than for the neutral items. These regions are very consistent with those shown in previous studies to be more predictive of successful encoding of emotional information than of neutral information (e.g., Kensinger \& Schacter, 2005a; Dolcos et al., 2004; reviewed by LaBar \& Cabeza, 2006).

Although it was heartening to replicate the standard subsequent-memory effects using a paradigm that differed from those previously employed to examine emotional memory, the main goals of this study were to move beyond an understanding of the regions that relate to memory for general information about a negative item's presentation (i.e., the ability to know whether or not a general type of negative item was studied) and to instead examine the neural processes that lead to effects of negative emotion on memory for detail. In particular, we had two main questions of interest. First, what neural processes correspond with a participant's ability to successfully encode the specific visual details of a presented negative item? Second, what are the neural mechanisms through which emotion exerts focal effects on memory? Analyses relevant to each of these questions are outlined below.

\section{Effects of Negative Emotion on Memory for Visual Detail}

We first conducted a conjunction analysis to examine the regions that corresponded with subsequent specific
Table 3. Regions in Which Neural Activity Was Greater during the Encoding of Items that Participants Would Later Remember ("Same" or "Similar" Responses to Same Items) than during the Encoding of Items that Participants Would Later Forget ("New" Responses to Same Items)

\begin{tabular}{|c|c|c|}
\hline Region & $\begin{array}{l}\text { Brodmann } \\
\text { Area }\end{array}$ & $\begin{array}{c}\text { Talairach } \\
\text { Coordinates } \\
(x, y, z)\end{array}$ \\
\hline \multicolumn{3}{|c|}{ Relation to Subsequent Memory for Negative and Neutral Item } \\
\hline Middle frontal gyrus & 10 & $30,56,17$ \\
\hline \multirow[t]{2}{*}{ Inferior temporal gyrus } & 37 & $-48,-59,-10$ \\
\hline & 37 & $53,-64,-4$ \\
\hline \multirow[t]{3}{*}{ Fusiform gyrus } & 37 & $-48,-52,-5$ \\
\hline & 37 & $27,-50,5$ \\
\hline & 20 & $21,-32,-14$ \\
\hline \multirow[t]{3}{*}{ Parahippocampal gyrus } & 36 & $-18,-7,-22$ \\
\hline & 36 & $-32,-8,-25$ \\
\hline & 36 & $-30,-8,-35$ \\
\hline \multirow[t]{3}{*}{ Hippocampus } & N/A & $36,-21,-20$ \\
\hline & & $36,-15,-17$ \\
\hline & & $-21,-9,-20$ \\
\hline \multirow[t]{2}{*}{ Striatum } & $\mathrm{N} / \mathrm{A}$ & $-18,3,-12$ \\
\hline & & $-21,5,-15$ \\
\hline \multicolumn{3}{|c|}{$\begin{array}{l}\text { Relation to Subsequent Memory for Negative but not for } \\
\text { Neutral Items }\end{array}$} \\
\hline Superior frontal gyrus & 8 & $-12,29,46$ \\
\hline $\begin{array}{l}\text { Inferior frontal gyrus } \\
\text { (orbito-frontal/ventrolateral) }\end{array}$ & $10 / 47$ & $-35,41,0$ \\
\hline Postcentral gyrus & 4 & $30,-6,28$ \\
\hline Inferior parietal lobe & 40 & $30,-37,25$ \\
\hline \multirow[t]{2}{*}{ Inferior temporal gyrus } & 37 & $56,-61,-2$ \\
\hline & 20 & $33,-2,-35$ \\
\hline \multirow[t]{3}{*}{ Fusiform gyrus } & $19 / 37$ & $-50,-67,-7$ \\
\hline & 37 & $-41,-56,-5$ \\
\hline & 38 & $-29,-36,-13$ \\
\hline Parahippocampal gyrus & 36 & $-30,-5,-33$ \\
\hline Hippocampus & $\mathrm{N} / \mathrm{A}$ & $38,-21,-14$ \\
\hline Amygdala & $\mathrm{N} / \mathrm{A}$ & $33,-4,-25$ \\
\hline \multirow[t]{3}{*}{ Striatum } & $\mathrm{N} / \mathrm{A}$ & $27,0,6$ \\
\hline & & $-3,7,16$ \\
\hline & & $6,-25,15$ \\
\hline Cerebellum & $\mathrm{N} / \mathrm{A}$ & $-32,-39,-21$ \\
\hline
\end{tabular}

All regions consist of at least 5 voxels. 
recognition for both negative and neutral items (i.e., regions in which activity was greater during the encoding of same items later given a "same" response than during the encoding of same items later given a "similar" response). To perform this conjunction analysis, we created a mask of the regions revealed in the contrast of specific $>$ nonspecific recognition of neutral items and then applied that mask to the contrast of specific $>$ nonspecific recognition of negative items. The individual contrasts included in the conjunction analysis were analyzed at a threshold of $p<.01$, uncorrected and with a 5-voxel extent (such that the conjoint probability of the conjunction analysis, using Fisher's estimate [Lazar et al., 2002; Fisher, 1950], was $p<.001$, uncorrected). Consistent with our hypothesis (and with results from Garoff et al., 2005 who investigated mnemonic specificity for neutral items), activity in the right fusiform gyrus (BA 37, Talairach coordinates: 34, $-42,-21)$ showed this correspondence to subsequent specific recognition. In addition, this analysis revealed that activity in the medial prefrontal cortex (BA 9, Talairach coordinates: $-4,45,32)$, in two regions of the left prefrontal cortex (BA 9/46, Talairach coordinates: $-48,36,18$; BA 8/9, Talairach coordinates: -42 , $20,41)$, and in a region of the right superior parietal lobule (BA 7, Talairach coordinates: 29, -65, 35) also was related to specific recognition for negative and neutral items (see red regions depicted in Figure 2). We extracted the signal change values from the right fusiform region (an ROI consisting of 23 voxels) and conducted an ANOVA with emotion type as a factor with two levels [negative arousing, neutral] and with memory specificity as a factor with three levels [later remembered with visual specificity ("same" response); later remembered with only general, nonspecific information ("similar" response); later forgotten ("new" response)]. This ANOVA revealed a main effect of memory specificity and an interaction between memory specificity and emotion $[F(2,18)=7.15, p<.05]$. The main effect of memory specificity reflected the fact that activity in this region of the right fusiform gyrus showed a correspondence to specific $>$ nonspecific recognition for both negative $[t(19)=3.83, p<.001]$ and neutral objects $[t(19)=2.01, p<.05]$, whereas it showed no correspondence to memory for nonspecific information (i.e., signal change for items later remembered with nonspecific detail was no greater than signal change for items later forgotten). However, as indicated by the significant interaction between emotion type and memory specificity, the relation of this region to specific recognition was stronger for the negative items than for the neutral items (see red bar graph in Figure 3). Thus, even in a region that was important for the encoding of specific visual detail for all items, there was a heightened correspondence to encoding specificity for the emotional items.

To examine whether there was a broader network of regions that would show this heightened association with
Figure 2. Encoding-related activity corresponding with subsequent specific recognition for all items (red regions), for negative items but not for neutral items (green regions), and for neutral items but not for negative items (blue regions). Signal changes from the regions of the fusiform gyrus outlined in yellow are depicted in Figure 3.

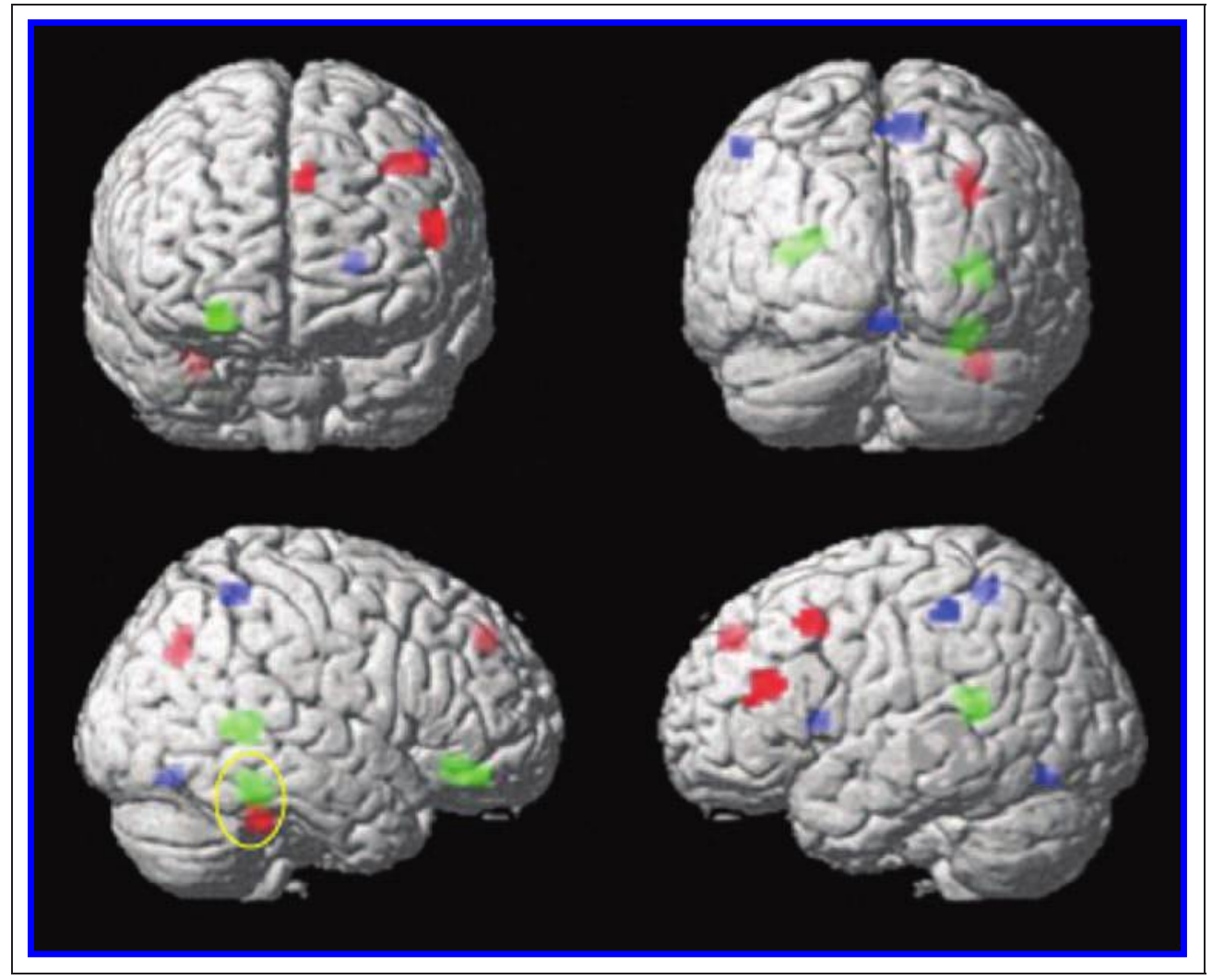




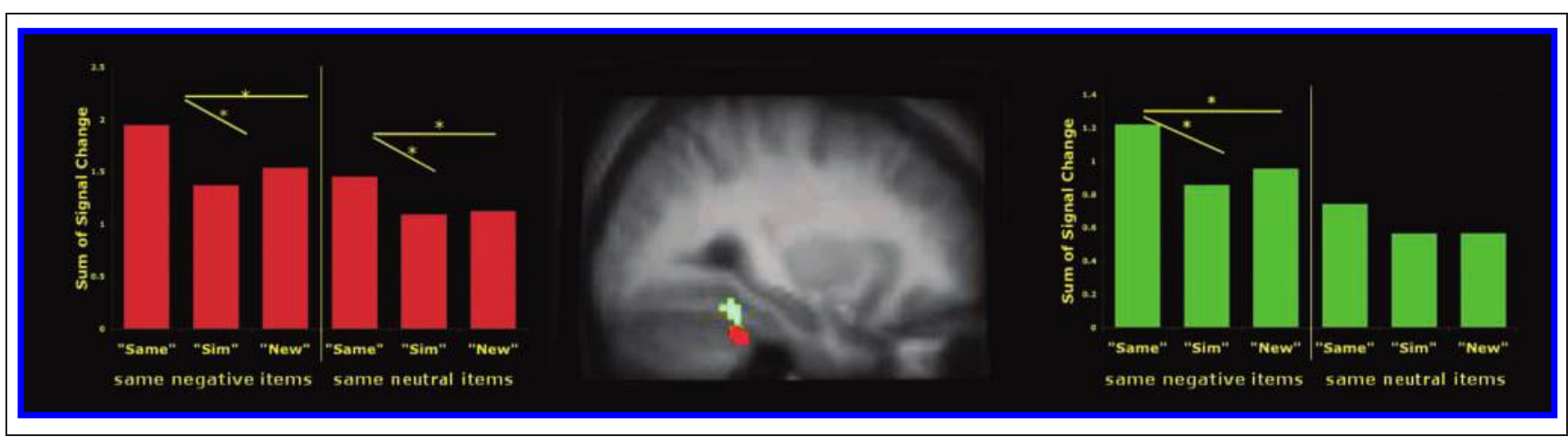

Figure 3. Signal changes in the regions of the right fusiform gyrus that were found to correspond with specific recognition for both negative and neutral items (depicted in red; Talairach coordinates: $34,-42,-21$ ) or with specific recognition only for negative items and not for neutral items (depicted in green; Talairach coordinates: 27, $-42,-15$ ). Bar graphs represent the signal change during the encoding of same items that were later recognized with specific visual detail ("same" response), recognized without specific visual detail ("similar" response), or completely forgotten ("new" response). Asterisks represent significant differences in signal change $(p<.05)$.

memory specificity for the emotional items, we performed a random-effects interaction analysis to reveal the regions that were more active during the processing of same emotional items that would later be recognized with specific visual detail than during the processing of same neutral items that would later be recognized with specific visual detail, that is, (specific $>$ nonspecific for negative) $>$ (specific $>$ nonspecific for neutral). At a threshold of $p<.001$, uncorrected and requiring a 5 -voxel extent of activation, this analysis revealed activity in a region of the right fusiform gyrus just medial and superior to the region that emerged in the conjunction analysis (BA 37, Talairach coordinates: $27,-42,-15$; see green region and bar graph in Figure 3, depicting activity from a 26-voxel ROI) as well as activity in the superior temporal gyrus (BA 22, Talairach coordinates: $33,-40$, 12) and in the orbito-frontal cortex (BA 11/47, Talairach coordinates: 26, 43, -15; green regions in Figure 2).

We also examined the regions that showed the reverse pattern of response: a greater correspondence to specific recognition for the neutral items than for the negative items. No regions within the fusiform gyrus were identified by this analysis; rather, the analysis revealed activity in the left inferior parietal lobe (BA 40, Talairach coordinates: $-51,-30,46)$, the precuneus (BA 7, Talairach coordinates: $6,-46,55)$, the striatum (Talairach coordinates: $-22,12,6$ ), and the cerebellum $(-1,-70,-10$; blue regions in Figure 2).

The results described above were consistent with our hypothesis that negative items are more likely to be remembered with visual detail than neutral items because of enhanced visual processing during encoding. We next wanted to examine whether there was evidence that interactions between the amygdala and the fusiform gyrus were important for this enhancement. To address this question, we defined the amygdala in an unbiased fashion, from a contrast comparing the processing of all items to the baseline fixation periods. A large region of the right amygdala was revealed in this contrast (see
Figure 4); SPM noted two distinct clusters of activation within this large swath of activity, a more ventral region (consisting of 26 voxels) and a more dorsal region (consisting of 17 voxels). Interestingly, there were no significant clusters of activity within the left amygdala at a standard threshold ( $p<.001$ and 5 voxels). There currently is much discussion about laterality effects in the amygdala, and it is not clear why amygdala activity in this study would be predominantly right-lateralized (see Kensinger \& Schacter, 2006b; Cahill, 2003, for further discussion). This laterality effect was consistent for men and women, and is consistent with right-lateralized activation shown in other studies that have used similar object stimuli (e.g., Kensinger \& Schacter, 2005a, 2005b).

To examine whether the right amygdala activity was related to subsequent memory for visual details, we conducted separate ANOVAs on the encoding-related signal change within each region of the right amygdala. We included subsequent memory specificity as a factor with three levels (later recognized the specific details of the same item ["same" response], later recognized only the general features of the same item ["similar" response], later forgotten ["new" response]), and emotion as a factor with two levels (negative, neutral). Critically, for each amygdalar region, this ANOVA revealed an interaction between memory specificity and emotion [for the ventral region, $F(2,18)=48.96, p<$ .001 ; for the dorsal region, $F(2,18)=32.57, p<.001]$. As can be seen in the solid yellow bar graph in Figure 4, both regions of the right amygdala showed greater activity during the encoding of negative items that later would be recognized with specific detail than during the encoding of negative items that later would be recognized with only general features [i.e., "same" > "similar," both $t(19)>5.5, p<.001] .{ }^{1}$ Both regions of the right amygdala also showed greater activity during the encoding of negative items that later would be recognized with at least general features than during the encoding of negative items that later would be forgotten [i.e., "similar" > 


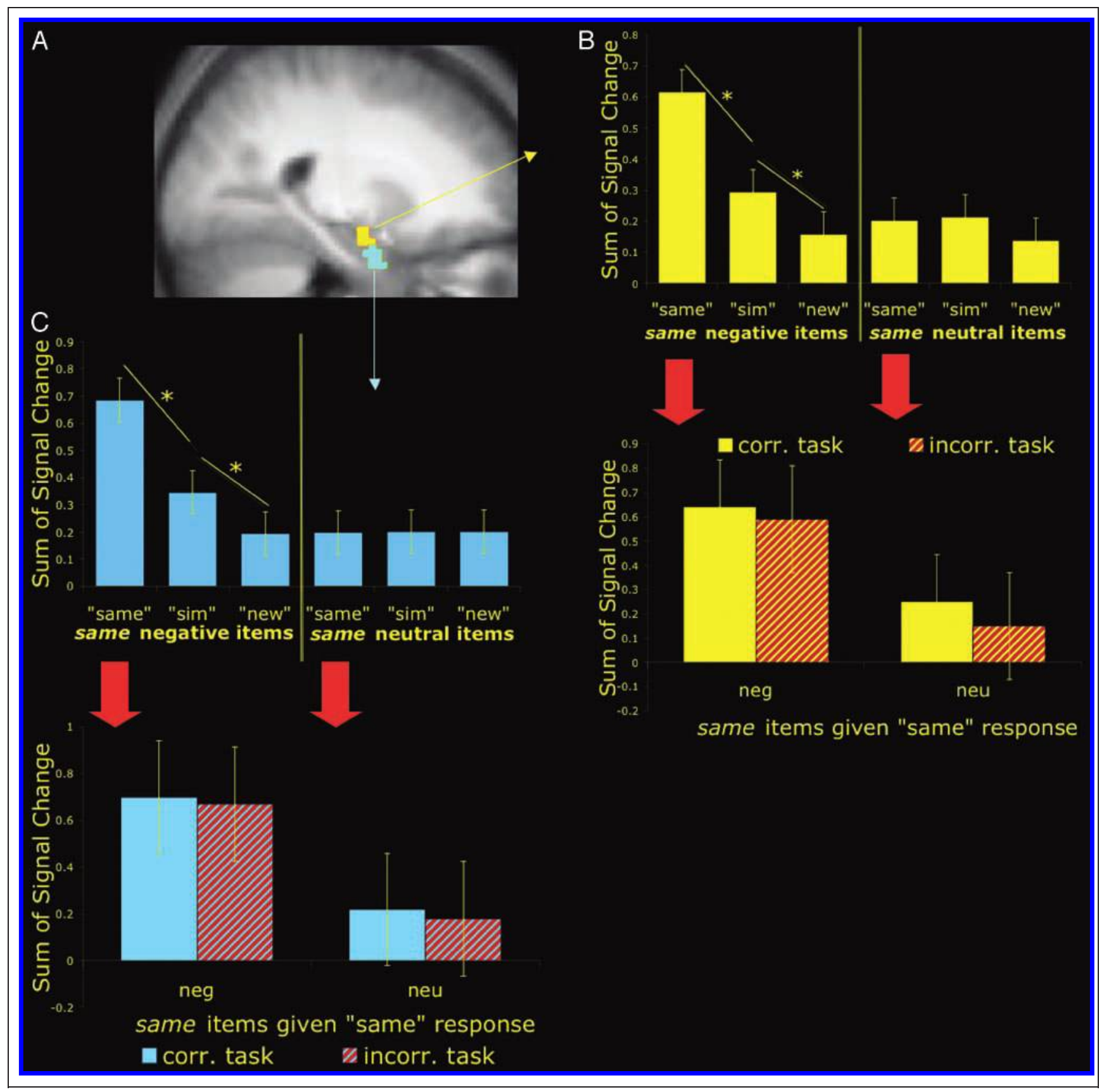

Figure 4. Regions of the amygdala were defined from the contrast of all images $>$ fixation. This analyses revealed two regions within the amygdala: One region in the right dorsal amygdala (region outlined in yellow; Talairach coordinates $21,-3,-12$ ) and one region in the right ventral amygdala (region outlined in blue; Talairach coordinates 24, 2, -25; panel A). As shown by the solid yellow (upper panel of B) and solid blue (upper panel of C) bar graphs, activity in both regions of the right amygdala corresponded with subsequent specific recognition of negative items: Activity was greater during the encoding of same negative items later given a "same" response than during the encoding of same negative items later given a "similar" response $(p<.001)$. Amygdala activity also corresponded with the likelihood of remembering at least the general features of a negative item (greater activity during encoding of negative items later given a "similar" response than during encoding of negative items later given a "new" response; $p<.001)$. Amygdala activity showed no relation to memory for the neutral items. In contrast to the relation between amygdala activity and the visual specificity of a memory (i.e., "same" > "similar" for negative items), amygdala activity showed no correspondence to memory for the decision made about a negative item. As shown in the comparison between the task-correct (solid bar) and task-incorrect (slashed bar) conditions (in the lower panels of B and C), activity in the amygdala was equally high for negative items remembered with or without the correct task assignment.

"new", both $t(19)>3.8, p<.001$; see Table 4 for all regions that showed this correspondence to "similar" > "new" responses to same items]. These findings are consistent with prior evidence that amygdala activity at encoding can correspond not only with the ability to remember that an item was previously presented (reviewed by Kensinger, in press; Hamann, 2001) but also with the ability to remember at least some details of that 
Table 4. Regions in Which Neural Activity Was Greater during the Encoding of Items that Participants Would Later Remember with General Item Features ("Similar" Response to Same Item) rather than Forget ("New" Response to Same Item)

\begin{tabular}{lcc}
\hline Region & $\begin{array}{c}\text { Brodmann's } \\
\text { Area }\end{array}$ & $\begin{array}{c}\text { Coordinates } \\
(x, y, z)\end{array}$ \\
\hline "Similar" > "New" for Negative and Neutral Items \\
Middle frontal gyrus & 6 & $36,4,30$ \\
"Similar" > "New" for Negative but not Neutral Items & $31,56,18$ \\
Middle frontal gyrus & 10 & $42,38,-2$ \\
Inferior frontal gyrus & 47 & $-48,36,-2$ \\
& 47 & $-21,40,-2$ \\
& $11 / 47$ & $48,7,14$ \\
Inferior parietal lobe & 44 & $27,-38,28$ \\
Lingual gyrus & $7 / 40$ & $-20,-81,10$ \\
Amygdala & 17 & $28,-4,-25$ \\
\hline
\end{tabular}

All regions consist of at least 5 voxels, active at a threshold of $p<.001$, uncorrected.

item's presentation (Kensinger \& Schacter, 2005a). In contrast, the right amygdala showed no correspondence to successful encoding of neutral items (all $p>.25$ ).

Although these results demonstrated a role for the amygdala in the encoding of visual specifics, we were interested in examining whether there was any evidence that this relation to memory for visually specific information stemmed from interactions between the amygdala and visual processing regions. To address this question, we computed correlations between the activity in the two regions of the right amygdala defined from the contrast of all $>$ fixation (i.e., the two regions in Figure 4) and activity in the right fusiform region that had been shown to be selectively implicated in the subsequent specific recognition of negative items (i.e., the fusiform region depicted in green in Figure 3). During the encoding of negative items that later were remembered with specific visual detail, robust correlations were revealed $(r=.67, p<.001$ in the dorsal amygdala; $r=.66, p<.01$ in the ventral amygdala; Figure 5). Importantly, no such correlations existed during the encoding of negative items that were forgotten $(p>.25)$, or during the encoding of any neutral items $(p>.25)$, and there was a significant difference in the strength of the correlations for the negative items that later were remembered with specific detail and for the other types of items (all Fisher's $z>2.32$, $p<.05)$. These results provided suggestive evidence for affective modulation of sensory processing: The individuals who showed the most robust amygdala activity during the visually specific encoding of the negative items also were the individuals who showed the most fusiform activity during the encoding of those items.

\section{Encoding Processes that Correspond with Focal Effects of Negative Emotion on Memory}

Our first question with regard to the focal effects of emotion on memory was whether amygdala activity would correspond with memory for any type of detail from the encoding episode, or only with memory for the intrinsic detail (the visual details of the object, as described above) and not with memory for an extrinsic detail (the decision made about the object at encoding). If amygdala activity enhances encoding of all event details, then not only should amygdala activity show a correspondence to subsequent memory for visual detail, it also should show a relation to subsequent memory for the task performed with the item. If, in contrast,

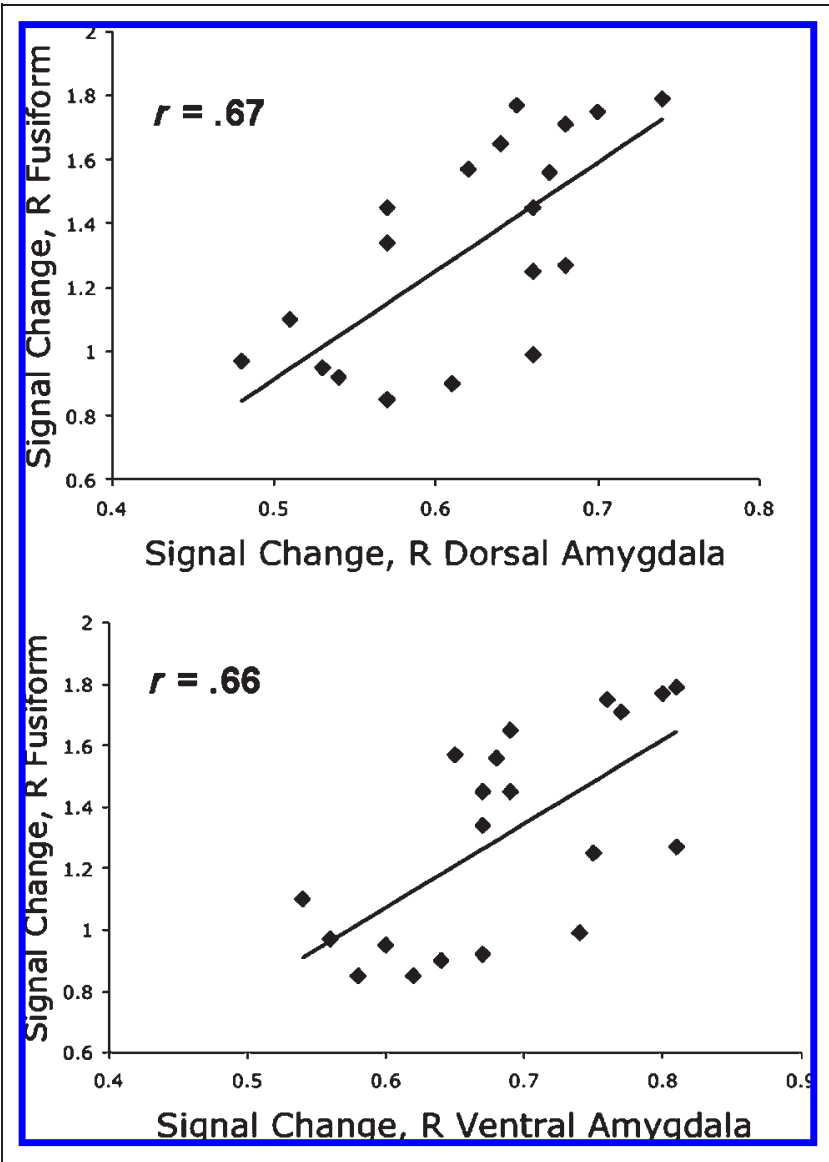

Figure 5. Activity in regions of the dorsal (top) and ventral (bottom) right amygdala correlated with activity in the right fusiform gyrus during the encoding of negative items that later would be remembered with specific visual detail. These results suggest that interactions between the amygdala and visual processing regions have important implications for the visual specificity with which negative information is remembered. 
amygdala activity primarily enhances a person's ability to remember details intrinsic to an item (such as its visual details), then the amygdala should show a selective relation to memory for visual details and not to memory for the task performed with an item.

To distinguish these alternatives, we computed the signal change, in the two regions of the right amygdala defined above, for negative and neutral items that were remembered with (a) both specific visual detail and task information (i.e., a "same" response to a same item and correct task selection), (b) specific visual detail but not task information (i.e., a "same" response to a same item and incorrect task selection), (c) task information but not specific detail (i.e., a "similar" response to a same item and correct task selection), and (d) neither task information nor specific visual detail (i.e., a "similar" response to a same item and incorrect task selection). We then conducted an ANOVA on these signal change values with visual specificity as a factor with two levels (visual specificity of memory present-i.e., "same" response given to same item; visual specificity of memory absent-i.e., "similar" response give to same item), task accuracy as a factor with two levels (task decision correct, task decision incorrect), and emotion type as a factor with two levels (negative arousing, neutral). This ANOVA revealed a significant effect of visual specificity $[F(1,19)=$ $31.01, p<.001$ for the dorsal region; $F(1,19)=30.67$, $p<.001$ for the ventral region] and an interaction between visual specificity and emotion $[F(1,19)=19.72, p<.001$ for the dorsal region; $F(1,19)=43.43, p<.001$ for the ventral region]. In contrast, neither region of the amygdala showed a main effect of task accuracy, nor an interaction between task accuracy and emotion $(p>.25)$. These results emphasize that although these regions of the right amygdala show a relation to memory for visual detail for negative arousing items, they show no correspondence to memory for the task performed with the negative arousing items. This pattern of results is made clear in Figure 4, in the bar graphs in the bottom of panels B and C. Although amygdala activity was greater for items later given a "same" response than for items later given a "similar" response (reflecting a correspondence to memory for visual detail), amygdala activity was no greater for items later attributed to the correct task than to items later attributed to the incorrect task. A whole-brain analysis confirmed that, when holding constant the level of visual detail later remembered about an item, no region of the amygdala was more active during the encoding of items later remembered with correct task information than during the encoding of items later remembered without correct task information; even when the threshold was dropped to $p<.05$, there was no evidence of a correspondence between amygdala activity and memory for task information. This result converges with prior findings (Kensinger \& Schacter, 2006a) in demonstrating that there are regions of the amygdala in which activity during encoding does not enhance memory for all details of an encoding episode.

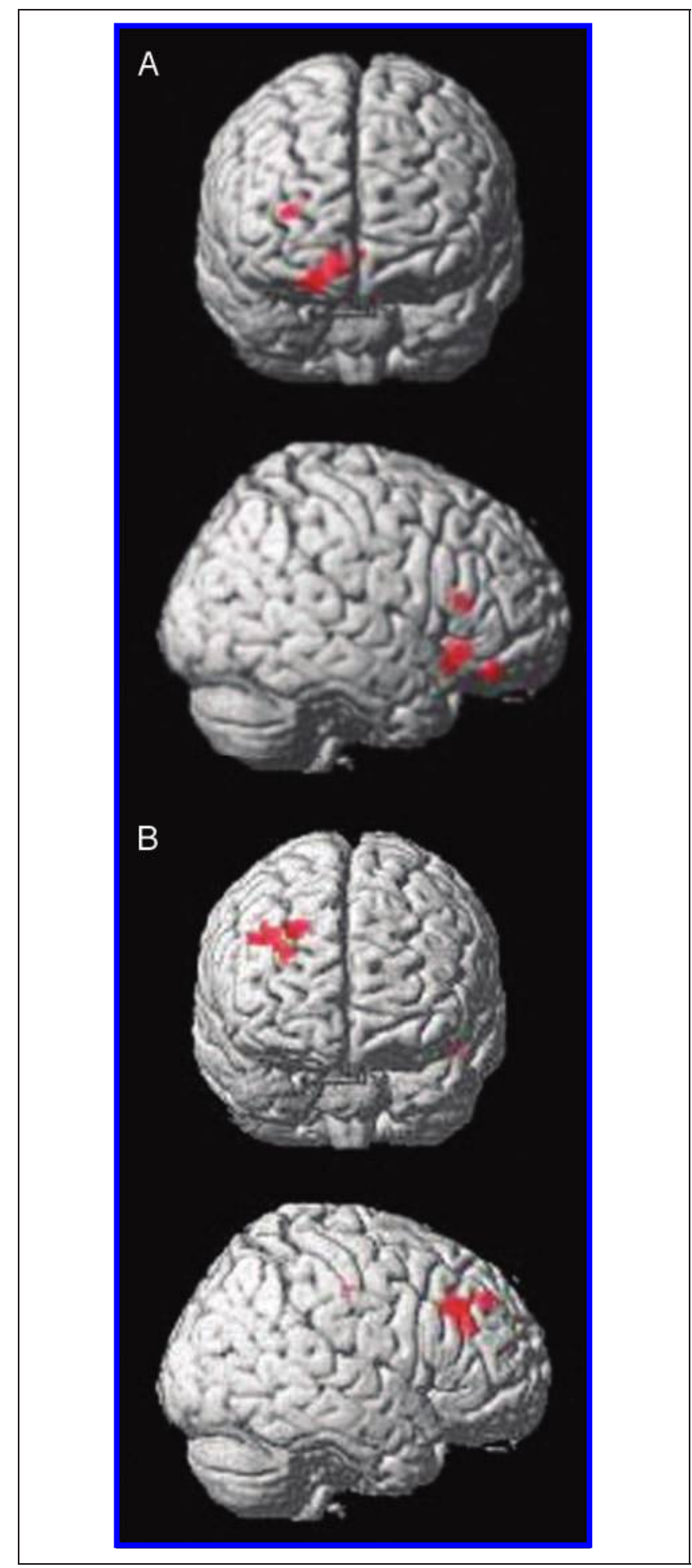

Figure 6. Activity in the right orbitofrontal cortex, anterior cingulate gyrus, and caudate nucleus corresponded with successful encoding of negative items' visual details and with unsuccessful encoding of the task performed with the negative items (A). A distinct network including the right dorsolateral prefrontal cortex, posterior cingulate gyrus, and left inferior temporal gyrus showed the same correspondence for neutral items (B).

To examine the network of regions that may lead to focal effects of emotion on memory (i.e., enhancing memory for some details but leaving memory for other details 
unaffected), we performed a conjunction analysis, using the masking function in SPM (as described above), to examine the encoding processes that led to successful encoding of the visual details of a negative item but not to successful encoding of the task performed with an item (i.e., subsequent specific $>$ nonspecific recognition AND task incorrect $>$ task correct for negative items only).

This conjunction analysis revealed activity in the orbito-frontal cortex (BA 10/11, Talairach coordinates: $20,58,-9)$, in the anterior cingulate gyrus (BA 24, Talairach coordinates: $11,23,-10)$, and in the caudate (Talairach coordinates: 18, 22, 4; Figure 6A). This result is consistent with the hypothesis that attentional processes at encoding serve to focus attention on the visual or intrinsic attributes of a negative item while diverting resources from processing information that is extrinsic to the item, such as the task being performed with the item.

The comparable conjunction analysis conducted for the neutral items revealed no regions when each contrast was analyzed with the standard threshold used for conjunction analyses $(p<.01)$. When the threshold of each contrast was lowered to $p<.05$, the analysis revealed a large region of the right dorsolateral prefrontal cortex (BA 9/46, Talairach coordinates: 40, 28, 30), the posterior cingulate gyrus (BA 24, Talairach coordinates: $15,-17,42)$, the left lateral parietal lobe (BA 40, Talairach coordinates: $-48,-28,46)$, and the lateral inferior temporal gyrus (BA 21, Talairach coordinates: $-50,-20,-14$; Figure 6B). Although these regions also are consistent with an attentional network, it is interesting to note that there is no overlap between the regions identified in the conjunction for the negative items and those revealed in the conjunction for the neutral items.

\section{DISCUSSION}

The primary goal of the present experiment was to assess the neural processes that underlie participants' enhanced ability to remember the visual details of a negative item. The secondary goal was to examine the neural mechanisms that lead to focal effects of emotion on memory for visual detail. With regard to the primary goal, the results indicate that this emotion-related enhancement in memory for visual detail results from enhancement in visual processing, particularly in the right fusiform gyrus. Activity in the right amygdala also corresponded with memory for visual detail, indicating that amygdala engagement can serve not only to increase memory for "gist" (see Adolphs et al., 2005 for further discussion) but also to enhance memory for visual detail. A strong correlation between right amygdala activity and right fusiform activity during the encoding of negative objects suggests an important contribution of interactions between these regions in modulating the effect of emotion on the visual specificity of object memory. In regard to the secondary goal, the results revealed that there are regions of the amygdala in which activity during encoding does not necessitate the successful encoding of all event details. Moreover, activity within the orbito-frontal cortex, anterior cingulate gyrus, and caudate nucleus corresponded with the ability to remember an item's visual detail but not the task performed with the item. This network of regions overlaps extensively with a network implicated in the detection of unattended emotional information (Vuilleumier, Armony, Driver, \& Dolan, 2001), suggesting that the relatively automatic or prioritized processing of emotional items may assist in the encoding of some intrinsic item attributes but may not benefit encoding of other, more extrinsic, details of an emotional item's presentation. Each of these findings will be expanded upon below.

\section{Emotion's Modulation of Memory for Visual Detail}

The present study provided evidence that the previously shown emotion-induced enhancement in memory for the exact visual details of an item (Kensinger et al., 2006) results from modulation of the same visual processes (mediated by the right fusiform gyrus) known to contribute to the specificity of visual priming effects (e.g., Koutstaal et al., 2001) and to the encoding and subsequent recognition of specific details of nonemotional items (Garoff et al., 2005). In particular, activity in the right fusiform gyrus corresponded with memory for the specific visual details of both negative and neutral items. Moreover, the right fusiform gyrus was more active, both in extent and in magnitude, during the encoding of negative items later recognized with specific visual detail than during the encoding of neutral items later recognized with specific detail. Thus, negative content seemed to enhance the visual processes that typically boost memory for specific information. Visual priming, encoding of nonemotional items, and encoding of emotional items depend, to some extent, on different underlying neural mechanisms, but our results highlight that engagement of the right fusiform contributes to each of these seemingly distinct effects. Based on this strengthening empirical foundation, an important task for future research is to identify precisely the right fusiform computations that contribute to visual memory specificity in different domains.

A strong correlation between the amount of activity in the right amygdala and in the right fusiform gyrus during the encoding of negative items later remembered with specific visual detail suggests that interactions between these regions may underlie this enhancement. There is abundant evidence that the amygdala can influence processing throughout the ventral visual processing stream, enhancing the processing of emotionally relevant stimuli (Sugase, Yamane, Ueno, \& Kawano, 1999; Amaral, Price, Pitkanen, \& Carmichael, 1992). Fusiform activity often is greater during the processing of fearful faces 
compared to neutral faces (Vuilleumier et al., 2001; Breiter et al., 1996; Morris et al., 1996), and correlations have been noted between the amount of activity in the amygdala and the amount of activity in the fusiform cortex during viewing of emotional information (Vuilleumier et al., 2001). Based on the speed of the anatomical connections between the amygdala and visual processing regions, there is reason to believe that at least part of the modulation occurs via the amygdala's influence on sensory processing (Amaral et al., 1992). Moreover, a neuroimaging study examining fusiform activation to fearful facial expressions in patients with varying amounts of damage to the amygdala showed a correlation between the amount of amygdalar preservation and the amount of fusiform activity (Vuilleumier et al., 2004).

Although the results of the present study cannot rule out the possible importance of fusiform modulation of amygdalar function, or of joint modulation of each of these regions by a third party, the results provide suggestive evidence that emotion's modulation of encoding processes can occur not only through interactions between the amygdala and the hippocampal formation (see Phelps, 2004, for a recent review) but also through interactions between the amygdala and sensory processing regions. Thus, amygdala-fusiform interactions, which have primarily been discussed within the context of emotion's ability to modulate attention and visual perception (see Dolan \& Vuilleumier, 2003; Davis \& Whalen, 2001) also may influence mnemonic performance. This conclusion makes functional sense given that items in the environment that are more likely to be detected and attended also will have an increased chance of being remembered (see Kensinger, 2004; Phelps, 2004, for recent reviews). The present results further suggest that this modulation of perceptual processing confers a particular advantage in remembering the exact visual features of an emotional item.

This conclusion fits nicely with the clinical literature examining memory in individuals who have experienced a traumatic event, such as being held up at gunpoint. Many of these individuals report incredibly vivid memories for salient aspects of the event, such as the details of the gun held by the perpetrator (see Kensinger et al., 2007; Reisberg \& Heuer, 2004). This study suggests that it may be amygdalar-fusiform interactions that underlie at least some of this boost in memory for the visual specifics of an emotional object in the environment.

\section{Neural Underpinnings of Negative Emotion's Focal Effects on Memory}

Although particular aspects of an event may be remembered with rich detail due to the event's negatively emotional content, negative emotion does not always enhance memory for all event details. Many researchers have discussed focal effects of emotion on memory in terms of tradeoffs (see Reisberg \& Heuer, 2004;
Buchanan \& Adolphs, 2002), whereby some aspects of an event are better remembered because of the event's emotional content, whereas other details are more likely to be forgotten. However, as demonstrated in the present study, there also are situations in which negative emotion appears to selectively enhance memory for some details while leaving memory for other episodic details relatively unaffected. In terms of the neural processes that lead to these focal effects of emotion on memory, the present study revealed that there are regions within the right amygdala that correspond with successful memory for some, but not all, details of a negative item's presentation. In particular, two regions of the right amygdala consistently were more active during the encoding of negative items that later would be remembered than during the encoding of negative items that later would be forgotten, and these regions also showed a relation to later memory for the visual details of the negative items' presentations. Activity in these right amygdalar regions, however, was unrelated to memory for the task performed with the negative items. Although it is possible that there are other regions of the amygdala, not revealed in the current study, that show a correspondence to successful encoding of a wider range of episodic details, these data emphasize that there are regions of the amygdala for which engagement does not lead to successful encoding of all details present during an encoding episode.

Note also that in the present paradigm, amygdala activity tracked with behavioral performance. Two regions within the right amygdala showed a subsequent-memory relation for the visual details of a negative item, a type of information that was more likely to be remembered when the item was negative than when it was neutral. In contrast, their activity did not correspond with later memory for the task performed with a negative item, a detail that was equally likely to be remembered for emotional and for neutral items. Future studies will be required to elucidate whether there tends to be a oneto-one correspondence between behavioral memory enhancement and amygdala engagement during encoding, or whether there are instances in which amygdala engagement modulates successful encoding of item details despite no overall effect of emotion on the likelihood of remembering that detail (and see Sharot et al., 2004 , for some evidence that amygdala activity can relate to memory performance even in the absence of overall memory-enhancing effects of emotion).

These data emphasize that activity within at least some regions of the amygdala can be differentially associated with memory for different types of details, enhancing memory for some aspects of an item's presentation while having no enhancing effect on memory for other aspects. But clearly, the amygdala is not acting in isolation. The present study revealed that activity in a network of regions previously implicated in the prioritized processing of emotional stimuli (Vuilleumier et al., 
2001) facilitated the encoding of a negative item's visual detail but did not enhance the encoding of the task performed with the item. The regions identified (orbitofrontal cortex, ventral striatum, and anterior cingulate gyrus) often are found to be brought on-line when a task requires engagement of motivational processes and of attention to affective stimuli (e.g., Everitt, Cardinal, Hall, Parkinson, \& Robbins, 2000; Schultz, Tremblay, \& Hollerman, 2000; Robbins \& Everitt, 1996). Thus, the correspondence of these regions to a selective enhancement in encoding of an intrinsic item attribute is consistent with the proposal that at least some of the focal effects of emotion on memory arise from the prioritized processing and attentional focusing on negative items during encoding. Attentional focusing on the item also can occur for nonemotional information, as suggested by the fact that activity in the dorsolateral prefrontal cortex, posterior cingulate gyrus, and lateral parietal lobule corresponded with memory for the visual specifics of, but not the task performed with, neutral items. However, the focus on emotional items appears to result through an affective-oriented guidance of attention rather than through other mechanisms of attention mediated by classic fronto-parietal networks (see Dolan \& Vuilleumier, 2003, for more discussion).

Although the exact mechanism through which this affective attention network exerts its effects remains to be specified, it is likely that activity in these regions modulates the functioning of other sensory or mnemonic processing systems (see Dolan \& Vuilleumier, 2003). A recent neuroimaging study examining retrieval of information studied in neutral or emotional contexts provided evidence that orbito-frontal activity could guide retrieval processes, modulating the strength of amygdalahippocampal connections (Smith, Stephan, Rugg, \& Dolan, 2006). It is plausible that just as the orbito-frontal cortex can guide retrieval processes, so might it focus and guide encoding processes.

\section{Conclusions}

In summary, the present results indicate that individuals are more likely to remember the visual details of negative items because of enhanced visual processing of those stimuli during encoding: The right fusiform gyrus showed enhanced activity, both in extent and in magnitude, during the encoding of negative items later remembered with visual detail. The results suggested that at least part of this modulation of visual processing stemmed from engagement of affective processing regions: Two regions of the right amygdala showed a strong correspondence to memory for a negative item's visual details, and their activity was correlated with activity in the right fusiform gyrus. Thus, emotion's modulation of encoding processes appears to occur not only through interactions between the amygdala and the hippocampal formation (see Phelps, 2004, for recent review) but also through interactions between the amygdala and sensory processing regions. Moreover, activity in an affective-attentional network (the orbitofrontal cortex, striatum, and anterior cingulate gyrus) corresponded with enhanced memory for the visual details of a negative item. This affective processing did not, however, lead to the successful encoding of all details of an item's presentation. Activity in these affective processing regions showed no correspondence (in the case of the amygdala) or a negative relation (in the case of the orbito-frontal cortex, striatum, and anterior cingulate gyrus) to the successful encoding of the task performed with an item. These results suggest that at least part of the reason for the focal effects of emotion on memory stem from encoding factors (see Reisberg \& Heuer, 2004): The more affectively focused individuals are on an emotional item, the more likely they are to remember intrinsic item attributes (such as the visual details of an item), but the less likely they are to remember details extrinsic to the item (such as the task performed with the item).

\section{Acknowledgments}

This research was supported by the National Institutes of Health grant MH60941 (to D. L. S.) and by the National Science Foundation (grant BCS0542694 to E. A. K. and a graduate research fellowship to R. J. G. E).

Reprint requests should be sent to Elizabeth A. Kensinger, McGuinn Hall, Room 510, 140 Commonwealth Ave., Chestnut Hill, MA 02467, or via e-mail: Elizabeth.kensinger@bc.edu.

\section{Note}

1. These ROI analyses revealed a correspondence between amygdala activity and visual specificity of memory for negative items but not for neutral items. Although, at a standard threshold, the whole-brain interaction analysis had not revealed amygdala activation, when the statistical threshold of that interaction analysis was lowered to $p<.05$, activation within the right dorsal amygdala (8 voxels) and ventral amygdala (6 voxels) was revealed. The pattern of activation within these regions paralleled those revealed in the dorsal and ventral amygdala ROIs, in terms of both overall signal change patterns and correlations with fusiform activity.

\section{REFERENCES}

Adolphs, R., Denburg, N. L., \& Tranel, D. (2001). The amygdala's role in long-term declarative memory for gist and detail. Behavioral Neuroscience, 115, 983-992.

Adolphs, R., Tranel, D., \& Buchanan, T. W. (2005). Amygdala damage impairs emotional memory for gist but not details of complex stimuli. Nature Neuroscience, 8 , 512-518.

Amaral, D. G., Price, J. L., Pitkanen, A., \& Carmichael, S. T. (1992). Anatomical organization of the primate amygdaloid complex. In J. Aggleton (Ed.), The amygdala: Neurobiological aspects of emotion, memory and mental dysfunction (pp. 1-66). New York: Wiley-Liss. 
Anderson, A. K., \& Phelps, E. A. (2001). Lesions of the human amygdala impair enhanced perception of emotionally salient events. Nature, 411, 305-309.

Breiter, H. C., Rauch, S. L., Kwong, K. K., Baker, J. R., Weisskoff, R. M., Kennedy, D. N., et al. (1996). Functional magnetic resonance imaging of symptom provocation in obsessive-compulsive disorder. Archives of General Psychiatry, 53, 595-606.

Buchanan, T. W., \& Adolphs, R. (2002). The role of the human amygdala in emotional modulation of long-term declarative memory. In S. Moore \& M. Oaksford (Eds.), Emotional cognition: From brain to behavior (pp. 9-34). Amsterdam: John Benjamins Publishing.

Cahill, L. (2003). Sex- and hemisphere-related influences on the neurobiology of emotionally influenced memory. Progress in Neuropsychopharmacology and Biological Psychiatry, 27, 1235-1241.

Dale, A. M. (1999). Optimal experimental design for event-related fMRI. Human Brain Mapping, 8, 109-114.

Davis, M., \& Whalen, P. J. (2001). The amygdala: Vigilance and emotion. Molecular Psvchiatrv, 6, 13-34.

Denburg, N. L., Buchanan, T. W., Tranel, D., \& Adolphs, R. (2003). Evidence for preserved emotional memory in normal older persons. Emotion, 3, 239-253.

Dewhurst, S. A., \& Parry, L. A. (2000). Emotionality, distinctiveness, and recollective experience. European Journal of Cognitive Psychology, 12, 541-551.

Dolan, R. J., \& Vuilleumier, P. (2003). Amygdala automaticity in emotional processing. Annals of the New York Academy of Sciences, 985, 348-355.

Dolcos, F., LaBar, K. S., \& Cabeza, R. (2004). Interaction between the amygdala and the medial temporal lobe memory system predicts better memory for emotional events. Neuron, 42, 855-863.

Everitt, B. J., Cardinal, R. N., Hall, J., Parkinson, J. A., \& Robbins, T. W. (2000). Differential involvement of amygdala subsystems in appetitive conditioning and drug addiction. In J. P. Aggleton (Ed.), The amygdala: A functional analysis (2nd ed., Chap. 10, pp. 353-359). Oxford: Oxford University Press.

Fisher, R. A. (1950). Statistical methods for research workers. London: Oliver and Boyd.

Garoff, R. J., Slotnick, S. D., \& Schacter, D. L. (2005). The neural origins of specific and general memory: The role of the fusiform cortex. Neuropsvchologia, 43, 847-859.

Hamann, S. (2001). Cognitive and neural mechanisms of emotional memory. Trends in Cognitive Sciences. 5 , 394-400.

Kensinger, E. A. (2004). Remembering emotional experiences: The contribution of valence and arousal. Reviews in the Neurosciences, 15, 241-251.

Kensinger, E. A. (in press). Neuroimaging the formation and retrieval of emotional memories. Brain mapping: New research. Hauppauge, NY: Nova Science Publishers.

Kensinger, E. A., \& Corkin, S. (2003). Memory enhancement for emotional words: Are emotional words more vividly remembered than neutral words? Memory \& Cognition, 31, 1169-1180.

Kensinger, E. A., Garoff-Eaton, R. J., \& Schacter, D. L. (2006). Memory for specific visual details can be enhanced by negative arousing content. Journal of Memory and Language, 54, 99-112.

Kensinger, E. A., Garoff-Eaton, R. J., \& Schacter, D. L. (2007). Effects of emotion on memory specificity: Memory trade-offs elicited by negative visually arousing stimuli. Journal of Memorv and Language, 56, 575-591.
Kensinger, E. A., \& Schacter, D. L. (2005a). Emotional content and reality-monitoring ability: fMRI evidence for the influence of encoding processes. Neurobsuchologia. 43, 1429-1443.

Kensinger, E. A., \& Schacter, D. L. (2005b). Retrieving accurate and distorted memories: Neuroimaging evidence for effects of emotion. Neuroimage, 27, 167-177.

Kensinger, E. A., \& Schacter, D. L. (2006a). Amygdala activity is associated with the successful encoding of item, but not source, information for positive and negative stimuli. Lournal of Neuroscience, 26, 2564-2570.

Kensinger, E. A., \& Schacter, D. L. (2006b). Reality monitoring and memory distortion: Effects of negative, arousing content. Memory \& Cognition, 34, 251-260.

Koutstaal, W., Wagner, A. D., Rotte, M., Maril, A., Buckner, R. L., \& Schacter, D. L. (2001). Perceptual specificity in visual object priming: fMRI evidence for a laterality difference in fusiform cortex. Neuropsychologia. 39, 184-199.

LaBar, K. S., \& Cabeza, R. (2006). Cognitive neuroscience of emotional memory. Nature Reviews Neuroscience, 7 , 54-64.

Lazar, N. A., Luna, B., Sweeney, J. A., \& Eddy, W. F. (2002). Combining brains: A survey of methods for statistical pooling of information. Neuroimage, 16, 538-550.

LeDoux, J. E. (1995). Emotion: Clues from the brain. Anmual Review of Psychology, 46, 209-235.

Marsolek, C. J. (1999). Dissociable neural subsystems underlie abstract and specific object recognition. Psychological Science, 10, 111-118.

Mather, M., Mitchell, K. J., Raye, C. L., Novak, D. L., Greene, E. J., \& Johnson, M. K. (2006). Emotional arousal can impair feature binding in working memory. Journal of Cognitive Neuroscience, 18, 614-625.

Morris, J. S., Frith, C. D., Perrett, D. I., Rowland, D., Young, A. W., Calder, A. J., et al. (1996). A differential neural response in the human amygdala to fearful and happy facial expressions. Nature, 31, 812-815.

Noesselt, T., Driver, J., Heinze, H. J., \& Dolan, R. (2005). Asymmetrical activation in the human brain during processing of fearful faces. Current Biology, 15, 424-429.

Ochsner, K. N. (2000). Are affective events richly "remembered" or simply familiar? The experience and process of recognizing feelings past. Journal of Experimental Psychology: General, 129, 242-261.

Ohman, A., Flykt, A., \& Esteves, F. (2001). Emotion drives attention: Detecting the snake in the grass. Journal of Experimental Psychology: General, 130, 466-478.

Paller, K. A., \& Wagner, A. D. (2002). Observing the transformation of experience into memory. Trends in Cognitive Science, 6, 93-102.

Phelps, E. A. (2004). Human emotion and memory: Interactions of the amygdala and hippocampal complex. Current Opinion in Neurobiology, 14, 198-202.

Phelps, E. A., Ling, S., \& Carrasco, M. (2006). Emotion facilitates perception and potentiates the perceptual benefits of attention. Psvchological Science, 17, 292-299.

Reisberg, D., \& Heuer, F. (2004). Remembering emotional events. In D. Reisberg \& P. Hertel (Eds.), Memory and emotion (pp. 3-41). New York: Oxford University Press.

Robbins, T. W., \& Everitt, B. J. (1996). Neurobehavioural mechanisms of reward and motivation. Current Opinion in Neurobiology, 6, 228-236.

Schacter, D. L., Dobbins, I. G., \& Schnyer, D. M. (2004). Specificity of priming: A cognitive neuroscience perspective. Nature Reviews Neuroscience, 5, 853-862.

Schacter, D. L., Gallo, D. A., \& Kensinger, E. A. (2007). The cognitive neuroscience of implicit and false memories: Perspectives on processing specificity. In J. S. Nairne (Ed.), 
The foundations of remembering: Essays in honor of Henry L. Roediger III (pp. 353-377). New York: Psychology Press. Schultz, W., Tremblay, L., \& Hollerman, J. R. (2000). Reward processing in primate orbitofrontal cortex and basal ganglia. Cerebral Cortex, 10, 272-284.

Sharot, T., Delgado, M. R., \& Phelps, E. A. (2004). How emotion enhances the feeling of remembering. Nature Neuroscience. 12, 1376-1380.

Simons, J. S., Koutstaal, W., Prince, S., Wagner, A. D., \& Schacter, D. L. (2003). Neural mechanisms of visual object priming: Evidence for perceptual and semantic distinctions in fusiform cortex. Neuroimage, 19, 613-626.

Smith, A. P., Stephan, K. E., Rugg, M. D., \& Dolan, R. J. (2006). Task and content modulate amygdala-hippocampal connectivity in emotional retrieval. Neuron, 49, 631-638.

Sugase, Y., Yamane, S., Ueno, S., \& Kawano, K. (1999). Global and fine information coded by single neurons in the temporal visual cortex. Nature, 400, 869-873.

Tabert, M. H., Borod, J. C., Tang, C. Y., Lange, G., Wei, T. C., Johnson, R., et al. (2001). Differential amygdala activation during emotional decision and recognition memory tasks using unpleasant words: An fMRI study. Neuropsychologia. 39, 556-573.

Talairach, J., \& Tournoux, P. (1988). Co-planar stereotaxic atlas of the buman brain. New York: Thieme Medical.

Vuilleumier, P., Armony, J. L., Driver, J., \& Dolan, R. J. (2001). Effects of attention and emotion on face processing in the human brain: An event-related fMRI study. Neuron, 30, 829-841.

Vuilleumier, P., Richardson, M. P., Armony, J. L., Driver, J., \& Dolan, R. J. (2004). Distinct influences of amygdala lesion on visual cortical activation during emotional face processing. Nature Neuroscience, 7, 1271-1278. 
This article has been cited by:

1. Elizabeth A. Kensinger, Daniel L. Schacter. 2008. Neural Processes Supporting Young and Older Adults' Emotional MemoriesNeural Processes Supporting Young and Older Adults' Emotional Memories. Journal of Cognitive Neuroscience 20:7, 1161-1173. [Abstract] [PDF] [PDF Plus]

2. C. Deruelle, B. Hubert, A. Santos, B. Wicker. 2008. Negative emotion does not enhance recall skills in adults with autistic spectrum disorders. Autism Research 1:2, 91-96. [CrossRef] 